\title{
Surveillance for hepatocellular carcinoma in a mixed- aetiology UK cohort with cirrhosis: does $\alpha$-fetoprotein still have a role?
}

\author{
Authors: Gwilym J Webb, ${ }^{A}$ Kathryn VC Wright, ${ }^{B}$ Elizabeth CB Harrod, ${ }^{C}$ David A Gorard, ${ }^{D}$ Jane D Collier ${ }^{E}$ \\ and Alexander KC Evans ${ }^{F}$
}

\begin{abstract}
Mortality from hepatocellular carcinoma (HCC) in people with cirrhosis is increasing whereas mortality from other causes is declining. Surveillance appears to reduce mortality but the optimal strategy is uncertain. Current guidelines differ by recommending ultrasonography alone or with $\alpha$-fetoprotein $(\alpha \mathrm{FP})$. Records in three UK hospitals were analysed from 2006 to 2011. Of 111 HCC cases identified, 24 (47.1\%) of those eligible were under surveillance: 21 (87.5\%) were under combined ultrasonography- $\alpha \mathrm{FP}, 2$ (8.3\%) ultrasonographyonly and 1 (4.2\%) $\alpha$ FP-only surveillance. $\alpha$ FP was elevated in $19(86.4 \%)$, and $\alpha \mathrm{FP}$ alone triggered a confirmatory study in 11 (9.9\%) overall and 7 (29.1\%) under surveillance. Surveillance, but not $\alpha \mathrm{FP}$, correlated with smaller tumours. Survival did not differ significantly between groups. Given that $\alpha$ FP use is associated with identifying smaller HCCs and that several diagnoses would have been delayed without $\alpha \mathrm{FP}$ in this real-life cohort, these data support ongoing $\alpha F P$ use. However, further work is necessary with regard to whether $\alpha$ FP translates into improved clinical outcome and overall cost effects. In our area, stopping $\alpha$ FP use would also represent $a$ significant change in practice.
\end{abstract}

KEYWORDS: Hepatocellular carcinoma, $\alpha F P$, ultrasonography

\section{Introduction}

Hepatocellular carcinoma (HCC) is the sixth most common cancer and the third most common cause of cancer-related

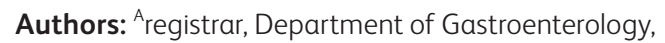
Wycombe Hospital, High Wycombe, UK, and Centre for Liver Research, Institute of Biomedical Research, University of Birmingham, Birmingham, UK; ${ }^{B}$ senior house officer, Department of Gastroenterology, John Radcliffe Hospital, Oxford, UK; 'senior house officer, Department of Gastroenterology, Royal Berkshire Hospital, Reading, UK; ${ }^{\text {D }}$ consultant, Department of

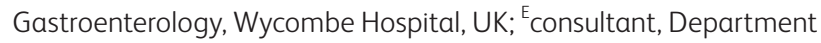
of Gastroenterology, John Radcliffe Hospital, UK; ' ${ }^{\text {c }}$ consultant, Department of Gastroenterology, Royal Berkshire Hospital, UK death worldwide ${ }^{1}$ and its incidence is continuing to rise. ${ }^{2}$ Cirrhosis is an established major risk factor for developing HCC, with HCC-associated mortality increasing in this population whereas mortality from other causes is declining. ${ }^{3}$ The overall prognosis for patients diagnosed with HCC is poor with an expected five-year survival rate of less than $5 \%$ across all patients. ${ }^{4}$

The use of surveillance programmes based on liver ultrasonography and measurement of serum $\alpha$-fetoprotein $(\alpha \mathrm{FP})$ is associated with identification of $\mathrm{HCC}$ at a smaller size and at an earlier clinical stage.$^{5,6}$ Early stage HCC has a wider range of therapeutic options and is potentially curable, whereas late-stage disease is not. ${ }^{7}$ In addition, early stage disease is associated with a greatly improved five-year survival rate of up to $70 \%{ }^{4}$. The use of ultrasonography and $\alpha \mathrm{FP}$ surveillance has been associated with decreased HCC-related mortality and longer survival among populations with chronic hepatitis B, some of whom had cirrhosis $;{ }^{8,9}$ studies of other at-risk groups are lacking.

Previously, a combination of ultrasonography and $\alpha$ FP-based surveillance has been recommended in groups at high risk of HCC. There has been a move away from $\alpha$ FP use towards ultrasonography alone on the basis that $\alpha \mathrm{FP}$ measurement has insufficient sensitivity and specificity and is poorly cost effective. ${ }^{7,10}$ However, ultrasonography's sensitivity and specificity for the identification of HCCs $<20 \mathrm{~mm}$ in size are reduced. ${ }^{11}$ Currently, the guidelines of the European Association for the Study of the Liver (EASL) and the American Association for the Study of Liver Diseases (AASLD) suggest surveillance based on ultrasonography alone, whereas British and Japanese guidelines continue to recommend surveillance based on the combination of $\alpha \mathrm{FP}$ and ultrasonography. ${ }^{12,13}$ Existing British guidelines were published in 2003 and so predate EASL (2012) and AASLD (2011) guidelines by several years. It is possible that, when British guidelines are updated, they may be altered to reflect the changes in approach recommended by others. However, recently there has been a return to the support of $\alpha \mathrm{FP}$ use in real-life surveillance programmes. ${ }^{2,14}$ This multicentre retrospective analysis aimed to assess the role of HCC surveillance, with particular reference to $\alpha \mathrm{FP}$, in British patients at elevated risk for HCC in real-life clinical practice. 


\section{Patients and methods}

Details of cases of HCC were retrospectively collected from the records of three British hospitals. Data were collected for a six-year period from two district general hospitals (Royal Berkshire Hospital, Reading and Wycombe Hospital, High Wycombe, 2006-2011 inclusive) and a four-year period from a tertiary referral centre (John Radcliffe Hospital, 2008-2011 inclusive). Together the hospitals serve a population of approximately $1,500,000$ people. A shorter study period was used for the tertiary centre because of incomplete availability of biochemical data before 2008. Cases were identified through the records of multidisciplinary meetings and clinical coding. Cases for which a diagnosis of HCC had been made on biopsy, imaging criteria or both were included. Data were obtained from hospital and general practitioner records.

Patient demographics, the presence of pre-established liver disease and participation in a HCC surveillance programme were recorded. In those undergoing surveillance, the surveillance modality was recorded: ultrasonography, combined ultrasonography- $\alpha \mathrm{FP}$ or $\alpha \mathrm{FP}$ alone. The assessment used to confirm HCC and its trigger, serum $\alpha F P$ levels at diagnosis and maximum HCC diameter, were also recorded. Date of diagnosis confirmation and, where available, date of death or survival to the end of the collection period (July 2013) were also obtained. Where the sole trigger for requesting a confirmatory test was an elevated $\alpha \mathrm{FP}$, this was recorded. In all hospitals, serum $\alpha F P$ was considered elevated when $\geq 8 \mathrm{ng} / \mathrm{ml}$. Statistical analysis was completed with Prism software (version 5.0b, GraphPad) using linear regression, and a MannWhitney-U test or Fisher's exact test as appropriate. Statistical significance was assumed when $\mathrm{p}<0.05$.

\section{Results}

The number of cases of HCC identified was 141. After the exclusion of 30 cases for which the available data were incomplete, 111 patients were analysed. Results are summarised in Table 1 and Fig 1.

Of the 111 patients diagnosed with HCC, 91 (82.0\%) patients were men, with a median age of 69 years (range 25-87).

Patients undergoing surveillance were younger than those not undergoing surveillance $(\mathrm{p}<0.0001)$, but no more likely to be of one gender than the other $(\mathrm{p}=0.556)$. Fifty-one $(45.9 \%, 38 \mathrm{men})$ patients had an existing diagnosis of chronic liver disease before the diagnosis of their HCC. Of patients with pre-established chronic liver disease, 24 (47.1\%) were undergoing regular surveillance for HCC. Of these, 21 (87.5\%) were undergoing combined ultrasonography- $\alpha \mathrm{FP}, 2$ (8.3\%) ultrasonographyonly and $1(4.2 \%) \alpha$ FP-only surveillance, respectively.

Table 1. Patient characteristics.

\begin{tabular}{|c|c|c|c|c|c|c|c|}
\hline & All patients & Percentage & Non-surveillance & Percentage & Surveillance & Percentage & p \\
\hline Total & 111 & 100 & 87 & 78.4 & 24 & 21.6 & N/A \\
\hline Men & 91 & 82.0 & 70 & 80.5 & 21 & 87.5 & 0.556 \\
\hline Women & 20 & 18.0 & 17 & 19.5 & 3 & 12.5 & 0.556 \\
\hline Median age, years (range) & \multicolumn{2}{|l|}{$69(25-87)$} & \multicolumn{2}{|l|}{$74(25-87)$} & \multicolumn{2}{|l|}{$59(46-82)$} & $<0.0001$ \\
\hline Prior liver disease & 51 & 45.9 & 27 & 31.0 & 24 & 100.0 & $<0.0001$ \\
\hline \multicolumn{8}{|l|}{ Aetiology ${ }^{a}$} \\
\hline Alcohol & 19 & 37.3 & 11 & 39.3 & 8 & 33.3 & 0.7753 \\
\hline Idiopathic & 6 & 11.8 & 3 & 10.7 & 3 & 12.5 & 1 \\
\hline NAFLD & 7 & 13.7 & 7 & 25.0 & 0 & 0.0 & 0.0114 \\
\hline HBV & 1 & 2.0 & 0 & 0.0 & 1 & 4.2 & 0.4615 \\
\hline $\mathrm{HCV}$ & 15 & 29.4 & 3 & 10.7 & 12 & 50.0 & 0.0024 \\
\hline PBC & 3 & 5.9 & 2 & 7.1 & 1 & 4.2 & 1 \\
\hline $\mathrm{HH}$ & 2 & 3.9 & 2 & 7.1 & 0 & 0.0 & 0.4932 \\
\hline$\alpha \mathrm{FP}$ measured at diagnosis & 104 & 93.7 & 80 & 92.0 & 24 & 100.0 & 0.583 \\
\hline$\alpha F P$ elevated at diagnosis & 81 & 77.9 & 61 & 76.3 & 20 & 83.3 & 0.299 \\
\hline $\begin{array}{l}\text { Median (range) } \alpha \mathrm{FP} \text { at } \\
\text { diagnosis, } \mathrm{ng} / \mathrm{ml}\end{array}$ & \multicolumn{2}{|c|}{$96(1-735,988)$} & \multicolumn{2}{|l|}{$100(1-735,988)$} & \multicolumn{2}{|l|}{$76(2-47,925)$} & 0.389 \\
\hline $\begin{array}{l}\text { Median (range) tumour size at } \\
\text { diagnosis, } \mathrm{mm}\end{array}$ & \multicolumn{2}{|l|}{$56(5-229)$} & \multicolumn{2}{|l|}{$70(5-229)$} & \multicolumn{2}{|l|}{$38.5(12-70)$} & 0.0007 \\
\hline Median survival, days (range) & \multicolumn{2}{|c|}{$258.5(5-1,820)$} & \multicolumn{2}{|l|}{$217.5(10-1,820)$} & \multicolumn{2}{|l|}{$404(5-1,393)$} & 0.5482 \\
\hline 1-year survival rate & \multicolumn{2}{|c|}{40 of $98(40.1 \%)$} & \multicolumn{2}{|l|}{29 of $78(37.2 \%)$} & \multicolumn{2}{|c|}{11 of $20(55 \%)$} & 0.2438 \\
\hline
\end{tabular}




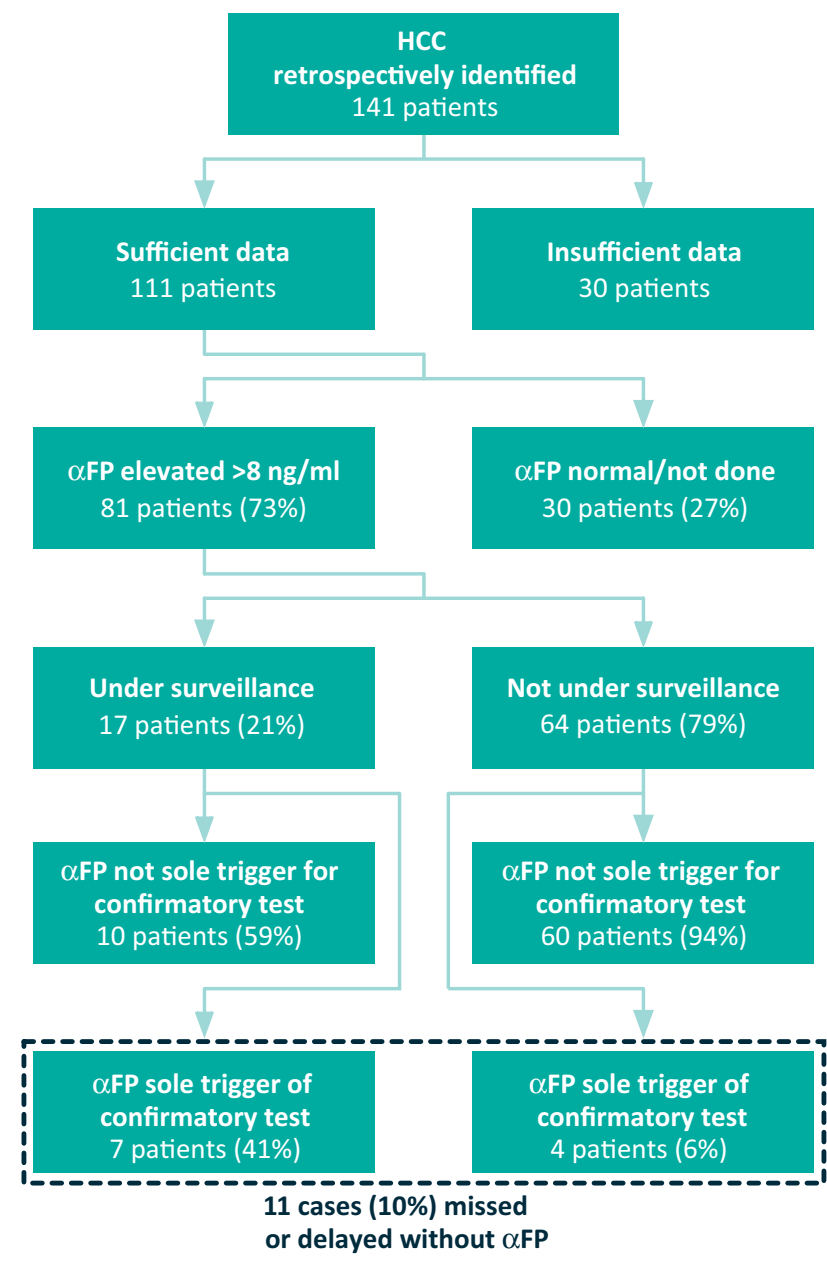

Fig 1. Usage of $\alpha \mathrm{FP}$ in HCC surveillance and diagnosis. $\alpha \mathrm{FP}=\alpha$-fetoprotein; $\mathrm{HCC}=$ hepatocellular carcinoma.

The diagnosis of HCC was confirmed by biopsy in 42 (37.8\%), computed tomography in 43 (38.7\%), magnetic resonance imaging in $25(22.5 \%)$ and ultrasonography combined with $\alpha \mathrm{FP}>200 \mathrm{ng} / \mathrm{ml}$ in $2(1.8 \%)$. At diagnosis, $\alpha \mathrm{FP}$ was elevated, ie $\geq 8 \mathrm{ng} / \mathrm{ml}$, in $77(69.4 \%)$, normal in $26(23.4 \%)$ and unmeasured in $8(7.2 \%)$ patients; the median $\alpha \mathrm{FP}$ was $96 \mathrm{ng} / \mathrm{ml}$ (range $1-735,988 \mathrm{ng} / \mathrm{ml})$. Of the $22(19.8 \%)$ patients who were diagnosed in an established surveillance programme using $\alpha \mathrm{FP}$, $19(86.4 \%)$ showed a rise in $\alpha \mathrm{FP}$ at the point of diagnosis.

When assessing the trigger for confirmatory cross-sectional imaging or biopsy across all patients, a solely elevated $\alpha \mathrm{FP}$ prompted further investigation in 11 (9.9\%); in those under surveillance, this number was 7 (29.1\%) with no abnormality detected on concurrent ultrasonography performed within the preceding three month period. Median $\alpha F P$ levels in these patients were $640 \mathrm{ng} / \mathrm{ml}$ (range 13-48,425 ng/ml) and $160 \mathrm{ng} /$ $\mathrm{ml}$ (range 13-1,648 ng/ml), respectively. Among those under surveillance, ultrasonography was suggestive of HCC in 70.9\% of cases and so triggered further investigation irrespective of $\alpha \mathrm{FP}$.

Tumour size was significantly smaller in those who were diagnosed as part of a surveillance programme (Fig 2).

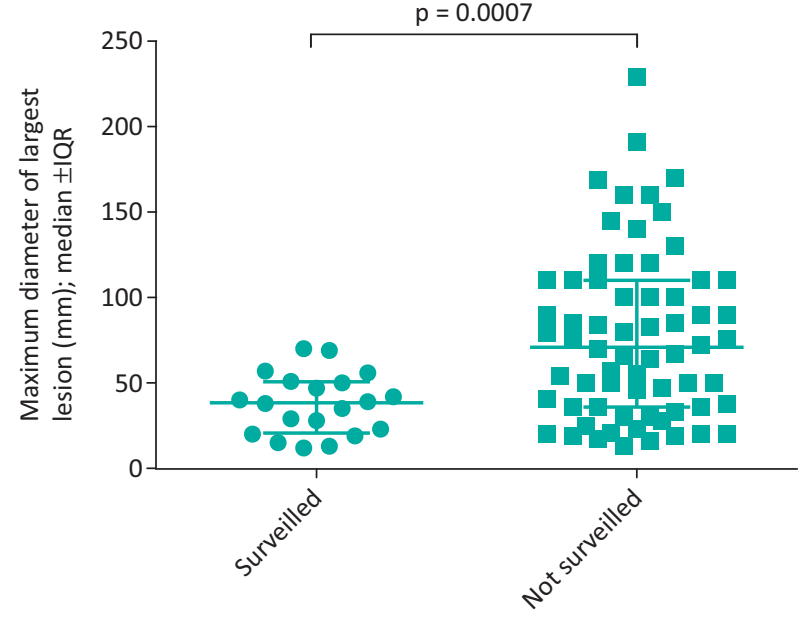

Fig 2. HCC maximal diameter at diagnosis within and outside surveillance. $\mathrm{HCC}=$ hepatocellular carcinoma; IQR, interquartile range.

Median tumour size was $70 \mathrm{~mm}$ (range 5-229 mm) outside surveillance and $38.5 \mathrm{~mm}$ (range within 12-70 $\mathrm{mm}$ ) within surveillance $(\mathrm{p}=0.0007)$; HCC size data were available for 76 of 111 patients $(68.4 \%)$. Tumour size did not correlate with $\alpha \mathrm{FP}$ when assessed among all HCCs $\left(\mathrm{R}^{2}=0.01994, \mathrm{p}=0.2367\right)$, or among cases with an elevated $\alpha \mathrm{FP}\left(\mathrm{R}^{2}=0.01316, \mathrm{p}=0.4180\right)$. Median tumour size was $36 \mathrm{~mm}$ (range 13-160 mm) among those with a normal $\alpha \mathrm{FP}$ and $57 \mathrm{~mm}$ (range 5-229 $\mathrm{mm}$ ) among those with an elevated $\alpha \mathrm{FP}(\mathrm{p}=0.0559)$. When assessing tumour size within the surveillance population, there was a trend towards those patients whose HCC was detected only by $\alpha$ FP measurement because they had smaller tumours, but this was not significant: median $39.5 \mathrm{~mm}$ vs $28.5 \mathrm{~mm}, \mathrm{p}=0.1879$.

Median tumour size was $46.5(12-160) \mathrm{mm}$ in surveillance vs 56.5 (5-229) mm outside ( $\mathrm{p}=0.56$ ); median survival did not differ significantly: $226(10-1,393)$ days vs $259.5(5-1,820)$ days. When the number of potentially curable tumours based on the Barcelona Clinic Liver Cancer (BCLC) criteria, ie those $<3 \mathrm{~cm}$, was assessed, 5 of 10 with data from the $\alpha \mathrm{FP}$ elevation-alone group, compared with 15 of 76 from the remainder, measured $<3 \mathrm{~cm}$ in maximum diameter $(\mathrm{p}=0.0481)$.

Reliable survival data were available for 98 of the 111 patients identified $(88.2 \%)$ and were gathered until the end of July 2013. Overall median survival was 258.5 (range $5-1,820$ ) days with 8 patients still alive at the end of the data collection period and a one-year survival of 40 out of 98 (40.8\%). Median survival in those who received surveillance of any form was 404 (range 5-1,393) days and in those who did not receive surveillance 217.50 (range $10-1,820$ ) days ( $\mathrm{p}=0.5482$ ). At one year, 11 out of 20 (55\%) of the surveillance group and 29 out of $78(37.2 \%)$ of the non-surveillance group had survived $(p=0.2026)$. When patients in whom a diagnosis was triggered by an elevated $\alpha$ FP level were compared with the remainder, the median survival was 226 and 258.5 days, respectively $(\mathrm{p}=0.6041)$. A survival plot of those under and those not under surveillance is presented in Fig 3. There was no significant difference between these groups using 


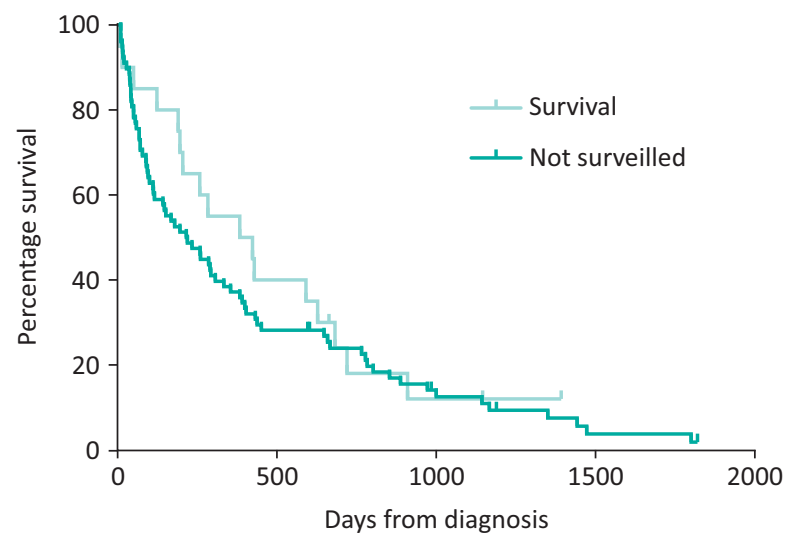

Fig 3. Survival of newly diagnosed patients within and outside hepatocellular surveillance programmes.

the Gehan-Breslow-Wilcoxon test $(\mathrm{p}=0.5562$; hazard ratio $=1.044)$. There was a non-significant trend towards size being linearly correlated to worsened survival $(\mathrm{p}=0.0834$, $\mathrm{R}^{2}=0.0461$; see Fig 4). The $\alpha \mathrm{FP}$ level did not correlate significantly with survival $\left(\mathrm{p}=0.4417, \mathrm{R}^{2}=0.0066\right)$.

\section{Discussion}

These results show that most cases of HCC diagnosed among our cohort were in individuals not undergoing regular HCC surveillance. This was despite almost a third of the patients with HCC carrying a diagnosis of chronic liver disease that would have made them eligible for surveillance. The low proportion of HCCs identified as part of a surveillance programme is consistent with other studies: $21.6 \%$ in our sample compared with $17 \%$ undergoing regular and $38 \%$ undergoing irregular surveillance in the largest published series, ${ }^{15}$ which is comparable with the $22 \%$ overall surveillance figure published in another British series. ${ }^{5}$

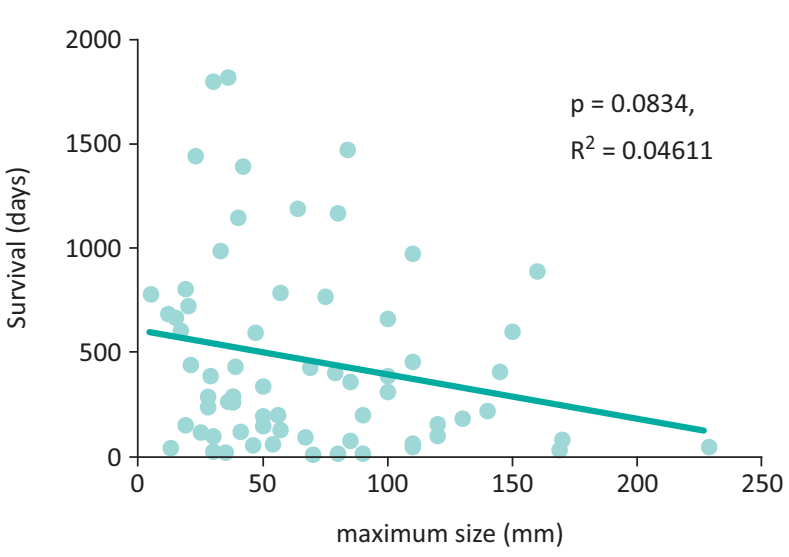

Fig 4. Survival versus $\mathrm{HCC}$ size across all patients. $\mathrm{HCC}=$ hepatocellular carcinoma.
Among our cohort of patients, those undergoing surveillance were younger. We speculate that clinicians preferentially put younger patients under surveillance because they were more likely to be eligible for curative treatment. It appeared that patients diagnosed with $\mathrm{HCC}$ and chronic hepatitis $\mathrm{C}$ (HCV) infection were more likely to be undergoing regular surveillance and those with non-alcoholic fatty liver disease (NAFLD) were less likely to be undergoing surveillance. The reason for this is not clear, but it may reflect a recognition of the elevated risk of HCC in HCV infection, that follow up may be more regular for other clinical reasons in HCV patients or that such patients may be younger; NAFLD patients may tend to be older with less regular clinical follow up.

In this series, which spanned clinical practice in three different centres and more than 20 consultant gastroenterologists/ hepatologists, of those under surveillance, $87.5 \%$ were receiving combined $\alpha \mathrm{FP}-$ ultrasonography surveillance. This is consistent with the older findings that this combination is favoured in the $\mathrm{UK}^{16}$ and with British guidelines, ${ }^{12}$ but is not consistent with the approach recommended by the newer international EASL and AASLD guidelines. We recognise that EASL and AASLD guidelines were updated only towards the end of our study period, so their effects on clinicians' practice may not yet be apparent.

Importantly, in our real-life series, a total of 7 (29.1\%) cases of HCC would have been likely to have a delay in their diagnosis if $\alpha \mathrm{FP}$ were not used in their surveillance strategy. This may be a result of the limitations of ultrasonography in these patients, including interoperator variability, variable patient body habitus, challenges in interpreting appearances in already nodular livers, variable attendance at ultrasonography and ultrasonography's reduced sensitivity for smaller lesions. ${ }^{17}$ The proportion of HCCs identified by ultrasonography in our surveillance group was $70.9 \%$. This may be compared with real-life reported sensitivities of ultrasonography in detecting HCC across all groups of $43.9 \%$ in a prospective study of patients attending a tertiary referral centre ${ }^{18}$ and a pooled sensitivity of an individual surveillance ultrasonography of $63 \%$ on meta-analysis. ${ }^{19}$ Our value of $70.9 \%$ is not a true sensitivity because it does not account for HCCs missed by both ultrasonography and $\alpha \mathrm{FP}$, and so will be falsely elevated.

Our study shows a non-significant trend towards HCCs that are diagnosed as a result of $\alpha \mathrm{FP}$ elevations alone being smaller than those detected with ultrasonography $\pm \alpha \mathrm{FP}$. We note that the cut-off $\alpha \mathrm{FP}$ used in the hospitals assessed in this study was relatively low at $8 \mathrm{ng} / \mathrm{ml}$. This contrasts with higher values of $20 \mathrm{ng} / \mathrm{ml}^{8,18}$ and $25 \mathrm{ng} / \mathrm{ml}^{9}$ used in other major studies, but is closer to a calculated optimum proposed by Paul et al of $10.7 \mathrm{ng} / \mathrm{ml} .{ }^{20}$ If a cut-off of $10.7 \mathrm{ng} / \mathrm{ml}$ had been used, no extra cases of HCC would have been missed or delayed; one case would have been missed with a cut-off of $20 \mathrm{ng} / \mathrm{ml}$ and two cases in total with $25 \mathrm{ng} / \mathrm{ml}$. The two studies referenced above that used higher cut-off values were investigating groups with viral hepatitis, who may have higher normal values of $\alpha \mathrm{FP} .{ }^{4}$ Current British guidelines do not define a surveillance $\alpha \mathrm{FP}$ cut-off or a specific assay. ${ }^{12} \mathrm{We}$ show that the use of $\alpha \mathrm{FP}$ in surveillance is associated with identification of HCC at a smaller size in routine British practice, and this is consistent with preliminary reports from elsewhere in Britain. ${ }^{5}$ 
We attempted to assess whether $\alpha$ FP levels correlated with tumour size but found no strong correlation among our cohort. In recognition of the fact that up to $20 \%$ of HCCs may not produce $\alpha \mathrm{FP},{ }^{12}$ we examined whether there was a correlation between $\alpha \mathrm{FP}$ and size when $\alpha \mathrm{FP}$ was elevated, and also found this to be non-significant ( $\mathrm{p}=0.2367$ ). These findings support the viewpoint that an elevated $\alpha \mathrm{FP}$ should be interpreted as a trigger for more definitive assessment with cross-sectional imaging rather than as a staging tool.

As mentioned above, smaller HCCs are generally associated with improved survival. ${ }^{4}$ In our study there was a nonsignificant trend in this direction. It is, however, unclear whether surveillance improves survival. Future trials assessing this would be difficult to conduct because surveillance of some form has become the standard of care. ${ }^{1}$ However, an improvement in survival has been suggested in both prospective and retrospective studies, both of which used the combination of $\alpha \mathrm{FP}$ and ultrasonography. ${ }^{8,9} \mathrm{~A}$ recent report on a prospective study examining a mixed cirrhotic population in Michigan, USA has reported that real-life surveillance performance, as assessed by improved sensitivity without a large decrease in specificity, is improved when $\alpha \mathrm{FP}$ is used in combination with ultrasonography. ${ }^{18}$ In our reallife, multi-centre UK study, we have not shown a significant survival advantage through the use of surveillance with or without $\alpha$ FP measurement, although there was a trend towards improved early survival; larger studies are needed. Our figures for median and one-year survival are comparable with those reported elsewhere. ${ }^{21}$ We have demonstrated that $\alpha \mathrm{FP}$ prevents a delay in diagnosis in a significant minority of patients. Importantly, therapies that improve the survival of early stage HCCs, such as radiofrequency ablation, transarterial chemoembolisation and the drug sorafenib, ${ }^{1}$ have become increasingly available during our study period and increase the importance of identifying HCCs earlier in their disease course. In our cohort, tumours were more likely to be potentially curable based on BCLC criteria in the $\alpha F P$-diagnosed cohort than the remainder.

As well as its benefit on survival being uncertain, a second concern about the use of $\alpha \mathrm{FP}$ in surveillance is the number of false positives generated. These generate anxiety for patients and extra cost for healthcare providers. Although this study was not designed to directly address this issue, at a single centre (Reading), results of all $\alpha \mathrm{FP}$ measurements performed for outpatient HCC surveillance were reviewed over two years - 2010-11. Of 499 taken, 45 (9.0\%) were $>8 \mathrm{ng} / \mathrm{ml}$. During this period, 22 new cases of HCC were diagnosed, giving a false-positive rate of $51 \%$ for this sub-section of our study population. Using our local estimated cost per $\alpha F P$ assay of $\mathfrak{E} 1.50$ gives an annual additional cost of $\mathfrak{E} 3 /$ patient. However, the substantially greater excess cost of $\alpha \mathrm{FP}$ is in triggering excess cross-sectional imaging studies. Were these results to be extrapolated to our population, an estimated 117 excess cross-sectional studies would probably have been generated, with the associated cost being several hundred pounds (approximately US $\$ 1,000$ ) per scan. Elsewhere, the estimated cost of using $\alpha \mathrm{FP}$ to find one tumour has been quoted at US $\$ 11,000-25,000 .^{22}$ Further work containing more detailed assessment of the health economic benefits of earlier detection is necessary to guide cost-directed recommendations about continued use of $\alpha \mathrm{FP}$ in surveillance programmes.

Our data suggest that, if $\alpha \mathrm{FP}$ measurement were removed from real-life HCC surveillance programmes, it would require a change in practice in our locality, and could result in delays in the diagnosis of a number of HCCs, ie a reduction in sensitivity; this may affect early survival, the likelihood of curative treatment being appropriate based on BCLC criteria. Further work is required to assess the specific role of $\alpha \mathrm{FP}$ measurement in real-life cohorts, to increase surveillance usage, to define an optimal practical $\alpha$ FP cut-off value should its continued use be recommended, and also to consider the number and cost of unnecessary cross-sectional imaging studies triggered by false-positive surveillance tests. The role of $\alpha$ FP in identifying HCCs earlier, so that patients may benefit from newer therapies, is unclear. Our work may be of relevance when UK HCC surveillance guidelines are reconsidered in British people with mixed-aetiology cirrhosis.

\section{References}

1 Forner A, Llovet JM, Bruix J. Hepatocellular carcinoma. Lancet 2012;379:1245-55.

2 El-Serag HB. Hepatocellular carcinoma. N Engl J Med 2011;365:1118-27.

3 Schuppan D, Afdhal NH. Liver cirrhosis. Lancet 2008;371:838-51.

4 El-Serag HB, Davila JA. Surveillance for hepatocellular carcinoma: in whom and how? Ther Adv Gastroenterol 2011;4:5-10.

5 Ahmed A, Mustafa Z, Neilson M et al. HCC diagnosed on surveillance programmes: impact on stage and outcome. Gut 2013;62:A83.

6 Trevisani F, Santi V, Gramenzi A et al. Surveillance for early diagnosis of hepatocellular carcinoma: is it effective in intermediate/ advanced cirrhosis? Am J Gastroenterol 2007;102:2448-57.

7 Bruix J, Sherman M. Management of hepatocellular carcinoma: An update. Hepatology 2011;53:1020-2.

8 Zhang B-H, Yang B-H, Tang Z-Y. Randomized controlled trial of screening for hepatocellular carcinoma. J Cancer Res Clin Oncol 2004;130:1-6.

9 McMahon BJ, Bulkow L, Harpster A et al. Screening for hepatocellular carcinoma in Alaska natives infected with chronic hepatitis B: a 16-year population-based study. Hepatology 2000;32:842-6.

10 EASL EAftSot, Cancer EOFRATO. EASL-EORTC clinical practice guidelines: Management of hepatocellular carcinoma. J Hepatol 2012;56:908-43.

11 Bolondi L, Gaiani S, Celli N et al. Characterization of small nodules in cirrhosis by assessment of vascularity: The problem of hypovascular hepatocellular carcinoma. Hepatology 2005;42:27-34.

12 Ryder S. Guidelines for the diagnosis and treatment of hepatocellular carcinoma (HCC) in adults. Gut 2003;52:iiil-iii8.

13 Japan Society of Hepatology. Surveillance algorithm and diagnostic algorithm for hepatocellular carcinoma. Hepatol Res 2010;40:6-7.

14 Giannini EG, Erroi V, Trevisani F. Effectiveness of alpha-fetoprotein for hepatocellular carcinoma surveillance: the return of the livingdead? Expert Rev Gastroenterol Hepatol 2012;6:441-4.

15 Davila JA, Morgan RO, Richardson PA et al. Use of surveillance for hepatocellular carcinoma among patients with cirrhosis in the United States. Hepatology 2010;52:132-41.

16 Lai C, Wardle M, Birkett V, Powell E, Thomas G. Surveillance for hepatocellular carcinoma in cirrhosis: a national audit of the practice in the UK. Gut 2002;50:a111-9.

17 Tong MJ, Blatt LM, Kao VW. Surveillance for hepatocellular carcinoma in patients with chronic viral hepatitis in the United States of America. J Gastroenterol Hepatol 2001;16:553-9. 
18 Singal AG, Conjeevaram HS, Volk ML et al. Effectiveness of hepatocellular carcinoma surveillance in patients with cirrhosis. Cancer Epidemiol Biomarkers Prev 2012;21:793-9.

19 Singal A, Volk ML, Waljee A et al. Meta-analysis: surveillance with ultrasound for early-stage hepatocellular carcinoma in patients with cirrhosis. Aliment Pharmacol Ther 2009;30:37-47.

20 Paul SB, Gulati MS, Sreenivas V et al. Evaluating patients with cirrhosis for hepatocellular carcinoma: value of clinical symptomatology, imaging and alpha-fetoprotein. Oncology 2007;72(suppl 1): $117-23$.
21 Altekruse SF, McGlynn KA, Reichman ME. Hepatocellular carcinoma incidence, mortality, and survival trends in the United States From 1975 to 2005. J Clin Oncol 2009;27:1485-91.

22 Bialecki E, Di Bisceglie A. Diagnosis of hepatocellular carcinoma. HPB (Oxford) 2005;7:26-34.

Address for correspondence: Dr AKC Evans, Department of Gastroenterology, Royal Berkshire Hospital, London Road, Reading RG1 5AN, UK.

Email:alex.evans@royalberkshire.nhs.uk

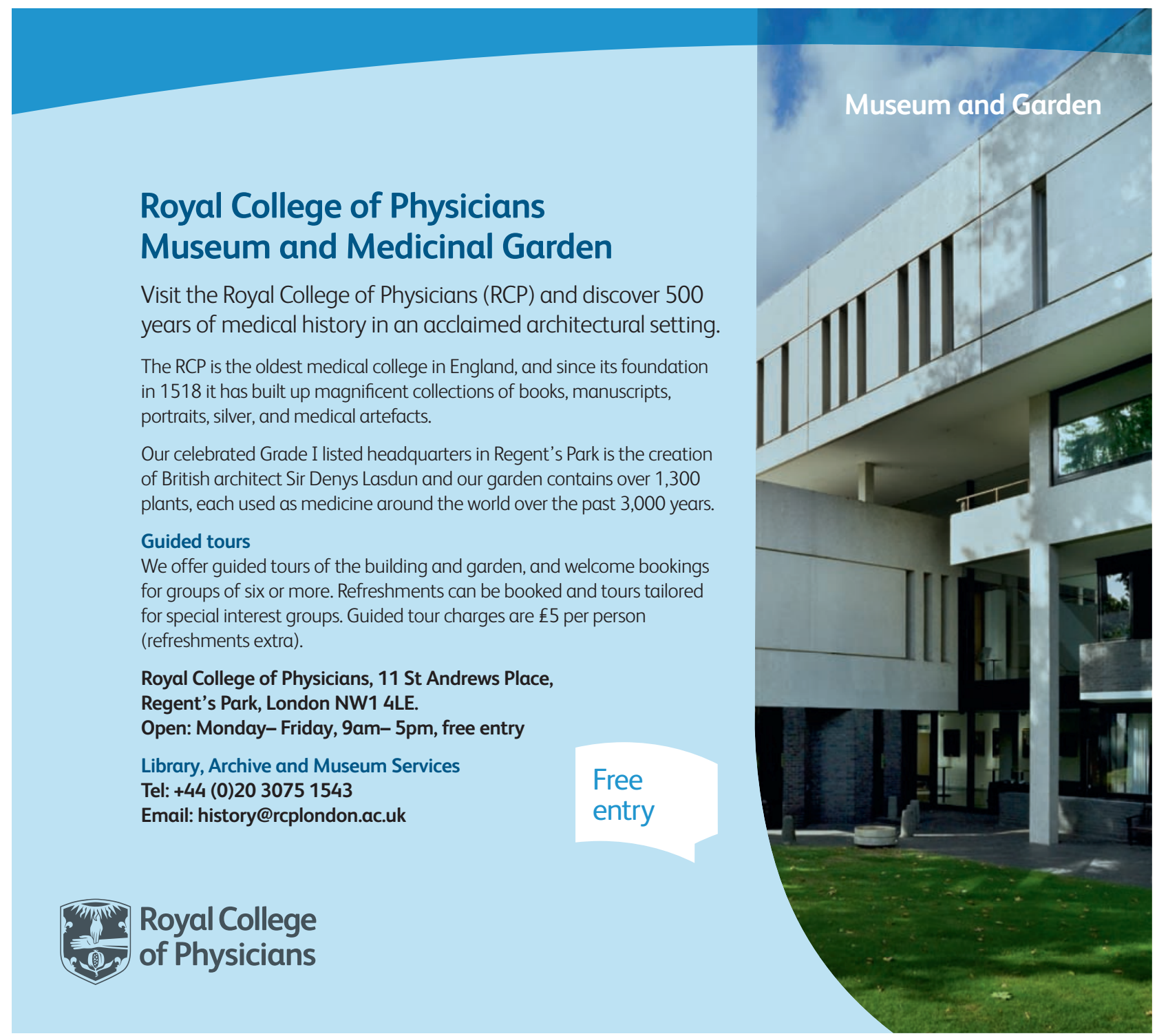

